\title{
The Validity of SOFA Score to Predict Mortality in Adult Patients with Cardiogenic Shock on Venoarterial Extracorporeal Membrane Oxygenation
}

\author{
Mohamed Laimoud $\mathbb{D}^{1,2}$ and Mosleh Alanazi ${ }^{1}$ \\ ${ }^{1}$ Adult Cardiac Surgical Intensive Care Unit (CSICU), King Faisal Specialist Hospital \& Research Center, Riyadh, Saudi Arabia \\ ${ }^{2}$ Critical Care Medicine Department, Cairo University, Cairo, Egypt \\ Correspondence should be addressed to Mohamed Laimoud; mlaimoud@kfshrc.edu.sa
}

Received 16 April 2020; Accepted 19 August 2020; Published 8 September 2020

Academic Editor: Samuel A. Tisherman

Copyright ( 92020 Mohamed Laimoud and Mosleh Alanazi. This is an open access article distributed under the Creative Commons Attribution License, which permits unrestricted use, distribution, and reproduction in any medium, provided the original work is properly cited.

Background. Venoarterial ECMO is increasingly used in resuscitation of adult patients with cardiogenic shock with variable mortality reports worldwide. Our objectives were to study the variables associated with hospital mortality in adult patients supported with VA-ECMO and to determine the validity of repeated assessments of those patients by the Sequential Organ Failure Assessment (SOFA) score for prediction of hospital mortality. We retrospectively studied adult patients admitted to the cardiac surgical critical care unit with cardiogenic shock supported with VA-ECMO from January 2015 to August 2019 in our tertiary care hospital. Results. One hundred and six patients supported with VA-ECMO were included in our study with in-hospital mortality of $56.6 \%$. The mean age of studied patients was $40.2 \pm 14.4$ years, and the patients were mostly males (69.8\%) with a mean BMI of $26.5 \pm 7$ without statistically significant differences between survivors and nonsurvivors. Presence of CKD, chronic atrial fibrillation, and cardiac surgeries was significantly more frequent in the nonsurvivors group. The nonsurvivors had more frequent AKI $(p<0.001)$, more haemodialysis use $(p<0.001)$, more gastrointestinal bleeding $(p=0.039)$, more ICH $(p=0.006)$, and fewer ICU days $(p=0.002)$ compared to the survivors group. The mean peak blood lactate level was $11 \pm 3$ vs $16.7 \pm 3.3, p<0.001$, and the mean lactate level after 24 hours of ECMO initiation was $2.2 \pm 0.9$ vs $7.9 \pm 5.7, p<0.001$, in the survivors and nonsurvivors, respectively. Initial SOFA score $\geq 13$ measured upon ICU admission had a $85 \%$ sensitivity and $73.9 \%$ specificity for predicting hospital mortality [AUROC $=0.862,95 \%$ CI: $0.791-0.932 ; p<0.001$ ] with $81 \%$ PPV, $79.1 \% \mathrm{NPV}$, and $80.2 \%$ accuracy while SOFA score $\geq 13$ at day 3 had $100 \%$ sensitivity and $91.3 \%$ specificity for predicting mortality with $93.8 \% \mathrm{PPV}, 100 \% \mathrm{NPV}$, and $96.2 \%$ accuracy [AUROC $=0.995,95 \%$ CI: $0.986-1 ; p<0.001$ ]. The $\Delta 1$ SOFA $(3-1) \geq 2$ had $95 \%$ sensitivity and $93.5 \%$ specificity for predicting hospital mortality [AUROC $=0.958,95 \%$ CI: $0.913-1 ; p<0.001$ ] with 95\% PPV, 93.5\% NPV, and $94.3 \%$ accuracy. SOFA score $\geq 15$ at day 5 had $98 \%$ sensitivity and $100 \%$ specificity for predicting mortality with $99 \%$ accuracy [AUROC $=0.994$, 95\% CI: $0.982-1 ; p<0.001]$. The $\Delta 2$ SOFA $(5-1) \geq 2$ had $90 \%$ sensitivity and $97.8 \%$ specificity for predicting hospital mortality [AUROC $=0.958,95 \%$ CI: 0.909-1; $p<0.001$ ] with 97.8\% PPV, 90\% NPV, and 94.8\% accuracy. Multivariable regression analysis revealed that increasing $\Delta 1$ SOFA score $(\mathrm{OR}=2.506,95 \% \mathrm{CI}: 1.681-3.735, p<0.001)$ and increasing blood lactate level $(\mathrm{OR}=1.388,95 \% \mathrm{CI}: 1.015-1.898, p=0.04)$ were significantly associated with hospital mortality after VA-ECMO support for adults with cardiogenic shock. Conclusion. The use of VA-ECMO in adult patients with cardiogenic shock is still associated with high mortality. Serial evaluation of those patients with SOFA score during the first few days of ECMO support is a good predictor of hospital mortality. Increase in SOFA score after 48 hours and hyperlactataemia are significantly associated with increased hospital mortality. 


\section{Background}

The reported mortality among adult patients with cardiogenic shock supported with VA-ECMO varies widely due to differences in patients background and clinical conditions [1-8]. The patients who undergo cardiac surgery may experience refractory postcardiotomy cardiogenic shock associated with increased morbidity and mortality. In these patients, VA-ECMO is considered as a rescue measure to achieve temporary circulatory and respiratory support allowing recovery of cardiac functions or bridging to further therapeutic options [9-14]. We conducted this study to detect the variables associated with hospital mortality in adult patients supported with VAECMO and to determine the validity of repeated assessments of those patients by SOFA score for prediction of hospital mortality.

\section{Methods}

2.1. Data Source. The study was approved by our institute ethics committee board, and the requirement for informed patient consent was waived because of the retrospective nature of the study. The Hospital Integrated Compliance Information System (ICIS) provided the database of our studied patients. The database included demographic, clinical, laboratory, and operative data, ICU and wards daily records, devices inserted, bedside procedures, and hospital discharge of all patients.

2.2. Patients Selection. We retrospectively evaluated consecutive patients who received VA-ECMO support between January 2015 and August 2019 at our tertiary care hospital. We enrolled patients $\geq 18$ years old in the study if they received VA-ECMO support for refractory cardiogenic shock. Exclusion criteria for patient selection were an age $<18$ years and veno-venous ECMO support.

2.3. ECMO Initiation and Patients Management. Venoarterial ECMO support was initiated by venous drainage from the femoral vein or right atrium with extracorporeal oxygen exchange and then return to the arterial system via the femoral artery (peripheral ECMO) or ascending aorta (central ECMO). In case of peripheral ECMO, an additional 6 Fr catheter was inserted distally into the femoral artery to avoid significant leg ischemia.

ECMO initiation and management were performed by trained ECMO team members. Daily checking of the ECMO oxygenator membrane and circuits was done by experienced perfusionists. After ECMO initiation, blood flow was adjusted based on clinical assessments including clearance of hyperlactatemia, mixed venous oxygen saturation, normalization of mean arterial blood pressure and urine output.

Checking of complete blood count and coagulation profile was routinely done for all patients before ECMO support. At ECMO initiation, a heparin bolus (intravenous 80 units/kg) was given, and then, continuous infusion with unfractionated heparin was maintained. The heparin bolus was not given if activated clotting time (ACT) was more than 300 seconds; then, serial measurements every hour were done till ACT was below 300 seconds and heparin infusion started. The heparin dose was adjusted according to the activated partial thromboplastin time (targeting 1.5-2-fold the normal-control value), AT III (goal 80-120\%), heparin assay (target $0.3-0.7$ units $/ \mathrm{ml}$ ), and clinical tolerance. Cryoprecipitate transfusions were used to keep fibrinogen more than $1 \mathrm{gm} / \mathrm{L}$, and platelets transfusions were used to keep count more than $50\left(10^{9} / \mathrm{L}\right)$.

2.4. The Studied Variables. Demographic, clinical, and laboratory data of studied patients were collected. The SOFA score was calculated on admission and then every 48 hours for 2 times. The worst values for each variable in the 24 hours period were used during score calculation. The assumed Glasgow Coma Scale (GCS) was used to assess the neurological status in studied patients. As part of our ICU policy, daily sedation vacation was done to assess the neurological state of patients including GCS assessment, any signs of lateralization, and brain stem reflexes. Urgent brain imaging with CT scanning was done within few hours in cases of delayed awakening or signs of lateralization. Regarding the coagulation component of SOFA score, we used the lowest platelet count before transfusions (if given). For the patients who developed renal impairment necessitating renal replacement therapy, we gave them a score of 4 for the renal component of SOFA score (Table 1).

The $\Delta$ SOFA score was the difference between 2 subsequent scores. The $\Delta 1$ SOFA score was the difference between SOFA score at day 3 and the admission score. The $\Delta 2$ SOFA score was the difference between SOFA score at day 5 and the admission scores [15-17].

2.5. Statistical Analysis. The patient characteristics were summarized using mean ( \pm standard deviation) or median (interquartile range (IQR)) for continuous variables and as frequency and percentage for categorical variables. Normality of data was checked using the Kolmogorov-Smirnov normality test. Comparisons between numerical data were done using Student's $t$-test or Mann-Whitney accordingly. Ordinal data were compared using the chi square test. $p$ values less than 0.05 were considered statistically significant. Assessment of the areas under the receiver operating characteristics (ROC) curves was performed. Logistic regression analysis was done to get the odds ratios (OR) and 95\% confidence intervals with hospital mortality as the dependent variable. Graphs were used to illustrate some information. Statistical tests were done using the Statistical Package of Social Science Software program, version 23 (SPSS).

\section{Results}

3.1. Baseline and Clinical Data. One hundred and six patients supported with VA-ECMO were included in our study after exclusion of pediatric cases and patients with $\mathrm{VV}$ ECMO. The in-hospital mortality was $56.6 \%$. The mean age 
TABLE 1: Criteria of the sequential organ failure assessment (SOFA) score [15-17].

\begin{tabular}{|c|c|c|c|c|c|}
\hline \multicolumn{6}{|c|}{ SOFA score } \\
\hline The variables & 0 & 1 & 2 & 3 & 4 \\
\hline $\begin{array}{l}\text { Respiratory system } \\
\mathrm{PaO}_{2} / \mathrm{FiO}_{2}(\mathrm{mmHg})\end{array}$ & $>400$ & $<400$ & $<300$ & $\begin{array}{c}<200 \text { with respiratory } \\
\text { support }\end{array}$ & $<100$ with respiratory support \\
\hline $\begin{array}{l}\text { Nervous system } \\
\text { Glasgow Coma Scale }\end{array}$ & 15 & $13-14$ & $10-12$ & $6-9$ & $<6$ \\
\hline $\begin{array}{l}\text { Cardiovascular system } \\
\text { Mean arterial pressure } \\
\text { (MAP) or administration } \\
\text { of vasopressors required }\end{array}$ & $\begin{array}{c}\text { MAP } \\
>70 \mathrm{mmHg}\end{array}$ & $\begin{array}{l}\text { MAP } \\
<70 \mathrm{~mm} / \\
\\
\mathrm{Hg}\end{array}$ & $\begin{array}{c}\text { Dopamine } \leq 5 \mu \mathrm{g} / \\
\mathrm{kg} / \mathrm{min} \text { or } \\
\text { dobutamine (any } \\
\text { dose) }\end{array}$ & $\begin{array}{c}\text { Dopamine }>5 \mu \mathrm{g} / \mathrm{kg} / \mathrm{min} \text { or } \\
\text { epinephrine } \leq 0.1 \mu \mathrm{g} / \mathrm{kg} / \mathrm{min} \\
\text { or norepinephrine } \leq 0.1 \mu \mathrm{g} / \\
\mathrm{kg} / \mathrm{min}\end{array}$ & $\begin{array}{c}\text { Dopamine }>15 \mu \mathrm{h} / \mathrm{kg} / \mathrm{min} \text { or } \\
\text { epinephrine }>0.1 \mu \mathrm{g} / \mathrm{kg} / \mathrm{min} \\
\text { or norepinephrine }>0.1 \mu \mathrm{g} / \\
\mathrm{kg} / \mathrm{min}\end{array}$ \\
\hline $\begin{array}{l}\text { Liver } \\
\text { Bilirubin } \\
(\mathrm{mg} / \mathrm{dl})(\mu \mathrm{mol} / \mathrm{L})\end{array}$ & $<1.2[<20]$ & $\begin{array}{l}1.2-1.9 \\
{[20-32]}\end{array}$ & $2.0-5.9[33-101]$ & $6.0-11.9[102-204]$ & $>12.0[>204]$ \\
\hline $\begin{array}{l}\text { Coagulation } \\
\text { Platelets } \times 10^{3} / \mathrm{ml}\end{array}$ & $>150$ & $<150$ & $<100$ & $<50$ & $<20$ \\
\hline $\begin{array}{l}\text { Kidneys } \\
\text { Creatinine }(\mathrm{mg} / \mathrm{dl}) \\
(\mu \mathrm{mol} / \mathrm{L}) \text {; urine output }\end{array}$ & $<1.2[<110]$ & $\begin{array}{c}1.2-1.9 \\
{[110-170]}\end{array}$ & $2.0-3.4[171-299]$ & $\begin{array}{l}3.5-4.9[300-440] \text { (or urine } \\
\text { output }<500 \mathrm{ml} / \text { day) }\end{array}$ & $\begin{array}{c}>5.0[>440] ; \text { urine output } \\
<200 \mathrm{ml} / \text { day }\end{array}$ \\
\hline
\end{tabular}

of studied patients was $40.2 \pm 14.4$ years, and the patients were mostly males (69.8\%) with a mean BMI of $26.5 \pm 7$ without statistically significant differences between survivors and nonsurvivors. Presence of chronic kidney disease was significantly more frequent in the nonsurvivors group $(p=0.001)$. Chronic atrial fibrillation $(p=0.006)$ and oral anticoagulation $(p=0.005)$ were statistically more frequent in the nonsurvivors group. There were no statistically significant differences between the survivors and nonsurvivors regarding underlying heart disease, diabetes mellitus, systemic hypertension, left ventricle EF, nor use of IABP. Cardiac surgeries were significantly more frequent in the nonsurvivors group but without significant differences regarding cardiopulmonary bypass or aortic cross clamping times. Central VA-ECMO was more frequent in the nonsurvivors, while peripheral VA-ECMO was more frequent in the survivors group $(p=0.006)$ without significant differences regarding ECMO days $(p=0.21)$. The nonsurvivors had more frequent AKI $(p \leq 0.001)$, more haemodialysis use $(p \leq 0.001)$, more GI bleeding $(p=0.039)$, more ICH $(p=0.006)$, and fewer ICU days $(p=0.002)$ as compared to the survivors group (Table 2).

3.2. Laboratory Data of Studied Patients. At ECMO initiation, the mean blood lactate level was $4.4 \pm 1.5$ vs $7.2 \pm 2$, $p<0.001$; the mean base excess was $-7.3 \pm 3.6$ vs $-10.4 \pm 3.1$, $p<0.001$, in the survivors and nonsurvivors, respectively. As compared to the survivors, the nonsurvivors had higher serum creatinine level $(p=0.018)$ without significant other laboratory differences (Table 3).

During ECMO support, the mean peak blood lactate level was $11 \pm 3$ vs $16.7 \pm 3.3, p<0.001$, and the mean lactate level after 24 hours of ECMO initiation was $2.2 \pm 0.9$ vs $7.9 \pm 5.7, p<0.001$, in the survivors and nonsurvivors, respectively. As compared to the survivors, the nonsurvivors had lower serum albumin level $(p=0.01)$ and higher serum creatinine $(p=0.017)$ and bilirubin levels $(p=0.03)$. The nonsurvivors had significant thrombocytopenia $(p<0.001)$ and lower aPTT and PTT ratio compared to the survivors (Table 4).

3.3. SOFA Scoring of Patients. The mean SOFA score at day 1 was $10.9 \pm 2.8$ vs $15.6 \pm 2.9, p<0.001$, the mean SOFA score at day 3 was $8.8 \pm 2.6$ vs $19.2 \pm 2.5, p<0.001$, and the mean SOFA score at day 5 was $7.6 \pm 2.2$ vs $19.8 \pm 2.5, p<0.001$, in the survivors and nonsurvivors, respectively. The median $\Delta 1$ SOFA (day 3-1) was $-2(-3$ to -1 ) vs $4(3-5), p<0.001$, and the median $\triangle 2$ SOFA (day $5-1$ ) was $-4(-5$ to -2 ) vs $4(3-6)$, $p<0.001$, in the survivors and nonsurvivors, respectively (Table 5 and Figure 1).

Initial SOFA score $\geq 13$ measured upon ICU admission had $85 \%$ sensitivity and $73.9 \%$ specificity for predicting hospital mortality [AUROC curve $=0.862$, 95\% CI: 0.791-0.932; $p<0.001]$ with 81\% PPV, 79.1\% NPV, and $80.2 \%$ accuracy while SOFA score $\geq 13$ at day 3 had $100 \%$ sensitivity and $91.3 \%$ specificity for predicting mortality with 93.8\% PPV, $100 \% \quad \mathrm{NPV}$, and $96.2 \%$ accuracy [AUROC $=0.995,95 \%$ CI: $0.986-1 ; p<0.001$ ]. The $\Delta 1$ SOFA (3-1) $\geq 2$ had $95 \%$ sensitivity and $93.5 \%$ specificity for predicting hospital mortality [AUROC $=0.958,95 \% \mathrm{CI}$ : $0.913-1 ; p<0.001]$ with 95\% PPV, 93.5\% NPV, and $94.3 \%$ accuracy. SOFA score $\geq 15$ at day 5 had $98 \%$ sensitivity and $100 \%$ specificity for predicting mortality with $99 \%$ accuracy [AUROC $=0.994,95 \%$ CI: $0.982-1 ; p<0.001$ ]. The $\Delta 2$ SOFA (5-1) $\geq 2$ had $90 \%$ sensitivity and $97.8 \%$ specificity for predicting hospital mortality [AUROC $=0.958,95 \% \mathrm{CI}$ : $0.909-1 ; p<0.001]$ with $97.8 \% \mathrm{PPV}, 90 \% \mathrm{NPV}$, and $94.8 \%$ accuracy (Table 6; Figures 2 and 3).

\section{Mortality Multivariable Analysis}

Multivariable regression analysis was done to get the odds ratios (OR) with 95\% confidence intervals (CI) with the hospital mortality as the dependent variable. Increasing $\Delta 1$ SOFA score $(\mathrm{OR}=2.506,95 \% \mathrm{CI}: 1.681-3.735, p<0.001)$ and progressive hyperlactatemia $(\mathrm{OR}=1.388,95 \% \mathrm{CI}$ : 1.015-1.898, $p=0.04$ ) were significantly associated with 
TABLE 2: Baseline characteristics of studied patients with VA-ECMO.

\begin{tabular}{|c|c|c|c|c|}
\hline Characteristics & All patients $(n=106)$ & Survivors $(n=46,43.4 \%)$ & Nonsurvivors $(n=60,56.6 \%)$ & $p$ value \\
\hline Age & $40.2 \pm 14.4$ & $39 \pm 10.9$ & $41.1 \pm 16.6$ & 0.73 \\
\hline \multicolumn{5}{|l|}{ Sex } \\
\hline Males & $74(69.8)$ & $31(67.4)$ & $43(71.7)$ & \multirow{2}{*}{0.61} \\
\hline Females & $32(30.2)$ & $15(32.6)$ & $17(28.3)$ & \\
\hline BMI & $26.5 \pm 7$ & $26.4 \pm 6.6$ & $26.6 \pm 7.4$ & 0.82 \\
\hline Diabetes mellitus & $20(18.9)$ & $9(19.6)$ & $11(18.3)$ & 0.84 \\
\hline Systemic hypertension & $34(32.1)$ & $14(30.4)$ & $20(33.3)$ & 0.75 \\
\hline CKD & $21(19.8)$ & $2(4.3)$ & $19(31.7)$ & 0.001 \\
\hline Pre-ECMO AF & $36(34)$ & $9(19.6)$ & $27(45)$ & 0.006 \\
\hline Anticoagulants intake & $39(36.8)$ & $10(21.7)$ & $29(48.3)$ & 0.005 \\
\hline LVEF\% & $29.5 \pm 13.8$ & $29.2 \pm 13.9$ & $29.6 \pm 13.9$ & 0.73 \\
\hline \multicolumn{5}{|l|}{ Heart disease } \\
\hline Dilated cardiomyopathy & $29(27.4)$ & $14(30.4)$ & $15(25)$ & \multirow{5}{*}{0.09} \\
\hline Ischemic cardiomyopathy & $26(24.5)$ & $15(32.6)$ & $11(18.3)$ & \\
\hline Valvular heart disease & $26(24.5)$ & $7(15.2)$ & $19(31.7)$ & \\
\hline $\mathrm{ACHD}$ & $5(4.7)$ & $1(2.2)$ & $4(6.7)$ & \\
\hline Others & $20(18.9)$ & $9(19.6)$ & $11(18.3)$ & \\
\hline Cardiac surgery & $61(57.5)$ & $21(45.7)$ & $40(66.7)$ & 0.03 \\
\hline \multicolumn{5}{|l|}{ Types of surgery } \\
\hline CABG & $3(4.9)$ & $2(9.5)$ & $1(2.5)$ & \multirow{6}{*}{0.16} \\
\hline Bentall operation & $9(14.7)$ & $3(14.3)$ & $6(15)$ & \\
\hline Valve surgery & $22(36.1)$ & $8(38.1)$ & $14(35)$ & \\
\hline CABG + valve surgery & $10(16.4)$ & $3(14.3)$ & $7(17.5)$ & \\
\hline Heart transplantation & $15(24.6)$ & $5(23.8)$ & $10(25)$ & \\
\hline Pulmonary endarterectomy & $2(3.3)$ & 0 & $2(5)$ & \\
\hline $\mathrm{CPB}$ time (minutes) & $231.7 \pm 93.5$ & $213.4 \pm 83.3$ & $239.9 \pm 97.6$ & 0.43 \\
\hline Aortic cross clamping (minutes) & $147.1 \pm 53$ & $144.3 \pm 51.7$ & $148.6 \pm 54.6$ & 0.85 \\
\hline IABP & $21(19.8)$ & $12(26.1)$ & $9(15)$ & 0.15 \\
\hline \multicolumn{5}{|l|}{ Type of ECMO } \\
\hline Central & $46(43.4)$ & $13(28.3)$ & $33(55)$ & \multirow{2}{*}{0.006} \\
\hline Peripheral & $60(56.6)$ & $33(71.7)$ & $27(45)$ & \\
\hline ECMO days & $9.7 \pm 7.2$ & $10.1 \pm 6.6$ & $9.5 \pm 7.7$ & 0.21 \\
\hline Upgrading to LVAD & $15(14.2)$ & $9(19.6)$ & $6(10)$ & 0.03 \\
\hline ICU days & $16.5(10-32)$ & $20(14-57)$ & $14(5.5-30.5)$ & 0.002 \\
\hline Post-ICU days & $16(10-25)$ & $16(10-25)$ & $10(4-16)$ & 0.23 \\
\hline Ventilator days & $10.5(6-26)$ & $9(8-26)$ & $13.5(5-25.5)$ & 0.95 \\
\hline AKI & $73(68.9)$ & $21(45.7)$ & $52(86.7)$ & $<0.001$ \\
\hline Haemodialysis & $50(47.2)$ & $9(19.6)$ & $41(68.3)$ & $<0.001$ \\
\hline Gastrointestinal bleeding & $24(22.6)$ & $6(13)$ & $18(30)$ & 0.039 \\
\hline Ischemic stroke & $13(12.3)$ & $3(6.5)$ & $10(16.7)$ & 0.11 \\
\hline Intracerebral haemorrhage & $13(12.3)$ & $1(2.2)$ & $12(20)$ & 0.006 \\
\hline
\end{tabular}

Data are presented mean $\pm \mathrm{SD}$, median (IQR), or $N(\%)$.

TABle 3: Laboratory data at ECMO insertion.

\begin{tabular}{|c|c|c|c|c|}
\hline Characteristics & All patients & Survivors & Nonsurvivors & $p$ value \\
\hline aPTT (seconds) & $51.3 \pm 26.1$ & $44.4 \pm 12.1$ & $56.7 \pm 32.2$ & 0.06 \\
\hline PTT ratio & $1.5 \pm 0.8$ & $1.3 \pm 0.4$ & $1.6 \pm 0.9$ & 0.14 \\
\hline INR & $1.7 \pm 0.5$ & $1.7 \pm 0.6$ & $1.7 \pm 0.4$ & 0.75 \\
\hline Fibrinogen $(\mathrm{g} / \mathrm{L})$ & $3.3 \pm 1.3$ & $3.5 \pm 1.4$ & $3 \pm 1.1$ & 0.24 \\
\hline Platelet count $\left(10^{9} / \mathrm{L}\right)$ & $164.5 \pm 90.8$ & $177.9 \pm 85.4$ & $154.2 \pm 94.2$ & 0.11 \\
\hline Haemoglobin $(\mathrm{gm} / \mathrm{L})$ & $114.5 \pm 19.8$ & $114.4 \pm 17.4$ & $114.6 \pm 21.6$ & 0.78 \\
\hline Base excess $(\mathrm{mmol} / \mathrm{L})$ & $-9 \pm 3.7$ & $-7.3 \pm 3.6$ & $-10.4 \pm 3.1$ & $<0.001$ \\
\hline Serum lactate $(\mathrm{mmol} / \mathrm{L})$ & $6 \pm 2.3$ & $4.4 \pm 1.5$ & $7.2 \pm 2$ & $<0.001$ \\
\hline Serum creatinine $(\mu \mathrm{mol} / \mathrm{L})$ & $112.9 \pm 64.9$ & $96.4 \pm 46$ & $125.5 \pm 74.2$ & 0.018 \\
\hline Serum bilirubin $(\mu \mathrm{mol} / \mathrm{L})$ & $30.3(18.9-58.9)$ & $28.6(15.7-58.7)$ & $37(22-61.7)$ & 0.09 \\
\hline
\end{tabular}

Data are presented mean \pm SD, median (IQR), or $N(\%)$. 
TABLE 4: Laboratory data during ECMO support.

\begin{tabular}{|c|c|c|c|c|}
\hline Characteristics & All patients & Survivors & Nonsurvivors & $p$ value \\
\hline aPTT (seconds) & $58.8 \pm 13.9$ & $60 \pm 9$ & $57.8 \pm 16.8$ & 0.02 \\
\hline PTT ratio & $1.7 \pm 0.4$ & $1.8 \pm 0.3$ & $1.7 \pm 0.5$ & 0.01 \\
\hline INR & $1.8 \pm 0.9$ & $1.6 \pm 0.4$ & $1.9 \pm 1.1$ & 0.09 \\
\hline Fibrinogen $(\mathrm{g} / \mathrm{L})$ & $3.3 \pm 1.5$ & $3.2 \pm 1.1$ & $3.3 \pm 1.8$ & 0.82 \\
\hline Platelet count $\left(10^{9} / \mathrm{L}\right)$ & $104 \pm 67$ & $135.1 \pm 55.3$ & $80.2 \pm 65.7$ & $<0.001$ \\
\hline Peak lactate level $(\mathrm{mmol} / \mathrm{L})$ & $14.2 \pm 4.3$ & $11 \pm 3$ & $16.7 \pm 3.3$ & $<0.001$ \\
\hline Lactate at 24 hours & $5.4 \pm 5.2$ & $2.2 \pm 0.9$ & $7.9 \pm 5.7$ & $<0.001$ \\
\hline Serum creatinine $(\mu \mathrm{mol} / \mathrm{L})$ & $139.2 \pm 66$ & $122.4 \pm 57.6$ & $151.9 \pm 69.5$ & 0.017 \\
\hline Serum bilirubin $(\mu \mathrm{mol} / \mathrm{L})$ & $84.1(48.7-183)$ & $78.3(34.9-172)$ & $84.3(60.3-267)$ & 0.03 \\
\hline Serum albumin $(g / L)$ & $32.8 \pm 5$ & $35.2 \pm 3.6$ & $31 \pm 5.2$ & 0.01 \\
\hline
\end{tabular}

Data are presented mean \pm SD, median (IQR), or $N$ (\%).

TABLE 5: SOFA scoring of studied VA-ECMO patients.

\begin{tabular}{lcccc}
\hline SOFA scores & $\begin{array}{c}\text { All } \\
\text { patients }\end{array}$ & Survivors & Nonsurvivors & $p$ value \\
\hline SOFA day 1 & $13.6 \pm 3.7$ & $10.9 \pm 2.8$ & $15.6 \pm 2.9$ & $<0.001$ \\
SOFA day 3 & $14.7 \pm 5.8$ & $8.8 \pm 2.6$ & $19.2 \pm 2.5$ & $<0.001$ \\
SOFA day 5 & $14 \pm 6.6$ & $7.6 \pm 2.2$ & $19.8 \pm 2.5$ & $<0.001$ \\
$\Delta$ 1 SOFA (day & $2(-2-4)$ & $-2(-3$ to & $4(3-5)$ & $<0.001$ \\
3-1) & $-1)$ & & \\
$\Delta$ 2 SOFA (day & $1(-4-4.5)$ & $-4(-5$ to & $4(3-6)$ & $<0.001$ \\
5-1) & $-2)$ &
\end{tabular}

Data are presented mean \pm SD or median (IQR).

hospital mortality after VA-ECMO support for adults with cardiogenic shock. Despite use of central VA-ECMO, haemodialysis, occurrence of ICH, and GI bleeding were significant in the nonsurvivors group, there were not significantly associated with mortality in the regression analysis (Table 7).

\section{Discussion}

We retrospectively analysed our adult patients with cardiogenic shock supported with VA-ECMO to detect the variables associated with hospital mortality in our tertiary care hospital. Our hospital mortality was $56.6 \%$, and most of our patients were males with a mean age of $40.2 \pm 14.4$ years. This finding is comparable to previous large studies, but our patients were younger than those in other studies.

Schmidt et al. extracted and analysed 3846 patients (mostly males with a median age of 54 years) with VAECMO use for refractory cardiogenic shock between the years of 2003 and 2013 from the Extracorporeal Life Support Organization (ELSO) registry and found only $42 \%$ survival at hospital discharge [18]. Maxwell et al. studied 8753 patients with ECMO use between the years 1998 and 2009 and found a mean age of $53.9 \pm 0.4$ years with $51 \%$ hospital mortality among all patients and a mean age of $48.9 \pm 0.8$ years for patients with cardiogenic shock with $64 \%$ hospital mortality [19].

El Sibai et al. retrospectively analysed 922 patients with cardiogenic shock on ECMO support from the US Nationwide Emergency Department Sample (NEDS) database and found that most of the patients were males with a mean age of 50.8 years and only $51 \%$ of them survived until hospital discharge [20]. Aso et al. analysed 5263 patients with VA-ECMO and found that about $73 \%$ of patients were males with a mean age of $64.8 \pm 13.7$ years and hospital mortality of $73.6 \%$ [21].

We studied the variables associated with hospital mortality and found that presence of CKD or development of AKI and use of renal replacement therapy were significant in the nonsurvivors patients. Schmidt et al. found renal failure as a significant variable in the mortality group and used it in creating the survival after venoarterial ECMO (SAVE) score [18]. Wang et al. found association of renal impairment and hospital mortality after CABG and VA-ECMO support and used the serum creatinine as one variable in calculating the REMEMBER score to predict mortality after CABG and VEECMO support [22]. However, Aso et al. found that renal impairment was not a significant variable, but the use of renal replacement therapy was significantly associated with mortality [21].

Most of our studied patients had refractory cardiogenic shock due to dilated or ischemic cardiomyopathy and valvular heart disease with about $57.5 \%$ of them as postcardiotomy shock. The proportion of adult congenital heart disease, myocarditis, and posttransplantation ECMO was small in our study. There were no significant differences between survivors and nonsurvivors regarding underlying heart disease, but the chronic AF and oral anticoagulation were significantly frequent in the nonsurvivors group. Schmidt et al. detected the better outcome of VA-ECMO for cardiogenic shock of patients with myocarditis or after heart or lung transplantation and the poor outcome of patients with congenital heart diseases, while there were no significant differences for ECMO support of cardiogenic shock due to valvular, ischemic, or other causes [18]. Combes et al. also described the better prognosis of VA-ECMO support for patients with myocarditis [6].

We noticed frequent use of central VA-ECMO in the nonsurvivors group and frequent use of peripheral VAECMO in the survivors. This may be related to the frequent cardiac surgeries and related complications like excessive bleeding and associated transfusions. However, central VAECMO use was not significant in the multivariate regression analysis model. Our results were going with Mariscalco et al. recent study of 718 adult patients with postcardiotomy shock 


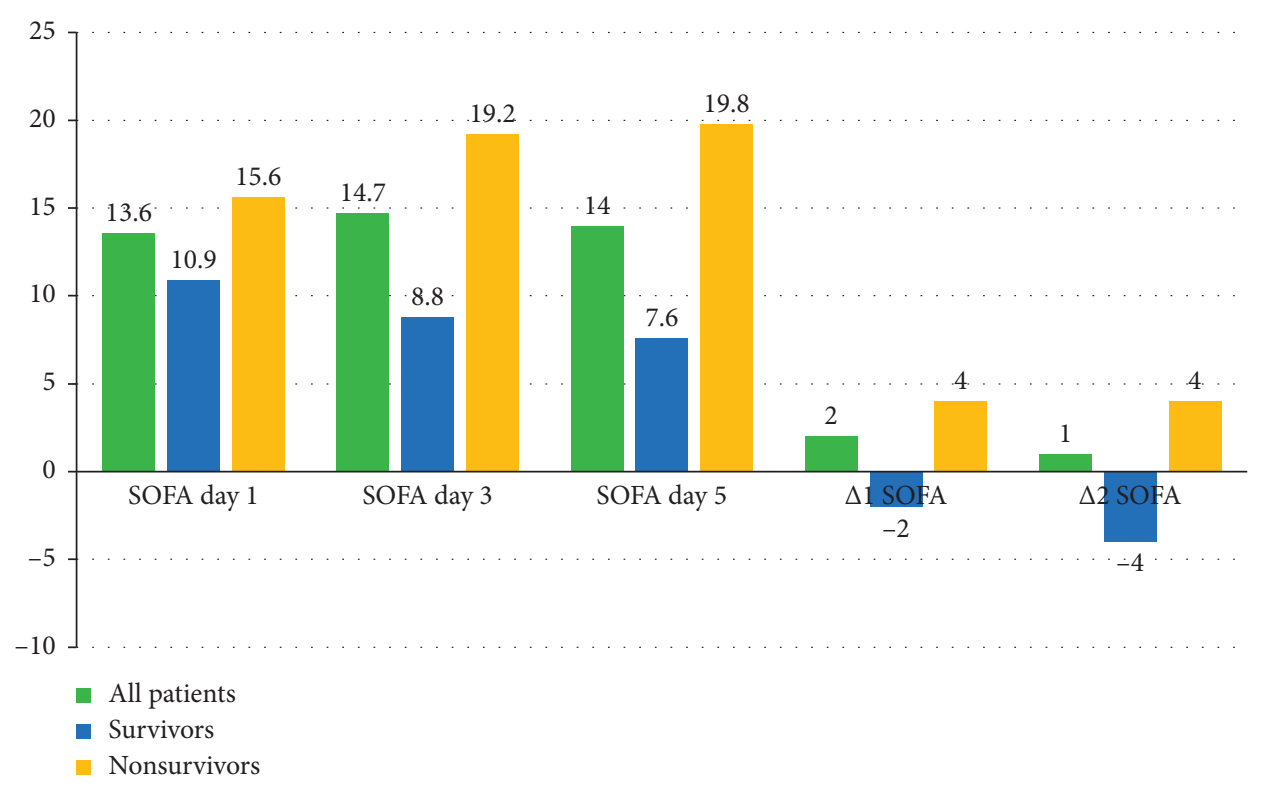

FIGURE 1: SOFA scoring of studied VA-ECMO patients.

TABle 6: The validity measures of SOFA scoring of studied VA-ECMO patients.

\begin{tabular}{lcccccccc}
\hline SOFA scores & AUROC & 95\% CI & Cut-off & Sensitivity (\%) & Specificity (\%) & PPV (\%) & NPV (\%) & Accuracy (\%) \\
\hline SOFA day 1 & 0.862 & $0.79-0.93$ & $\geq 13$ & 85.0 & 73.9 & 81.0 & 79.1 & 80.2 \\
SOFA day 3 & 0.995 & $0.986-1.0$ & $\geq 13$ & 100.0 & 91.3 & 93.8 & 100 & 96.2 \\
SOFA day 5 & 0.994 & $0.982-1.0$ & $\geq 15$ & 98.0 & 100.0 & 100 & 97.9 & 99.0 \\
$\Delta$ 1 SOFA (3-1) & 0.958 & $0.913-1.0$ & $\geq 2$ & 95.0 & 93.5 & 95.0 & 93.5 & 94.3 \\
$\Delta$ 2 SOFA (5-1) & 0.958 & $0.909-1.0$ & $\geq 2$ & 90.0 & 97.8 & 97.8 & 90.0 & 94.8 \\
\hline
\end{tabular}

AUROC: area under ROC; CI: confidence interval; PPV: positive predictive value; NPV: negative predictive value.

on VA-ECMO support from 19 cardiac surgical centers. Mariscalco et al. concluded that central VA-ECMO was associated with more hospital mortality, more bleeding, and excess blood products transfusions compared to peripheral VA-ECMO [23]. On the contrary, Djordjevic et al. retrospectively analysed 156 patients with postcardiotomy cardiogenic shock on VA-ECMO support and excluded significant 30 days mortality differences between central and peripheral VA-ECMO groups, but the patients with peripheral ECMO had significantly less mediastinal bleeding, less fresh frozen plasma transfusions, and less mediastinal explorations compared to those with central VA-ECMO support [24].

It is recognized that VA-ECMO decreases the coronary blood flow and increases left ventricular afterload, while IABP could reduce these effects and theoretically promote myocardial recovery and survival $[25,26]$. Our results showed absence of significant mortality difference with the concomitant use of IABP and VA-ECMO. This finding is consistent with Schmidt et al.'s study [18] but different from Aso et al.'s [21] study that detected lower mortality with IABP use. Moreover, Wang et al. did not find mortality difference with IABP use either before or after VA-ECMO insertion [22].

The occurrence of gastrointestinal and intracerebral bleeding was significantly frequent in the nonsurvivors group in the univariate analysis but was insignificant in the multivariate regression analysis model. They could be related to the significant coagulopathy and thrombocytopenia or as a part of multiorgan system failure.

Our results showed significantly higher arterial lactate level and greater metabolic acidosis at ECMO initiation in the nonsurvivors group compared to the survivors. Also, the lactate level reached a higher peak with delayed clearance in the nonsurvivors. Hyperlactatemia was significantly associated with mortality in our multivariable regression model $(\mathrm{OR}=1.388,95 \% \mathrm{CI}: 1.015-1.898, p=0.04)$. Schmidt et al. [18] described the significant metabolic acidosis in the nonsurvivors and detected the association of pre-ECMO serum $\mathrm{HCO}_{3} \leq 15 \mathrm{mmol} / \mathrm{L}$ and mortality $(\mathrm{OR}=0.7,95 \% \mathrm{CI}$ : 0.58-0.83, $p<0.0001)$. Moreover, Chen et al. [27] described the greater metabolic acidosis and hyperlactatemia in the nonsurvivors during urgent VA-ECMO insertion for cardiac and noncardiac causes at emergency department and used lactate level to develop the modified SAVE score. The impact of hyperlactatemia on mortality has been reported in adult patients with cardiogenic shock and VA-ECMO support $[28,29]$.

The use of VA-ECMO especially for refractory cardiogenic shock is a complex bundle of associated measures such as invasive mechanical ventilation and anticoagulation with possible blood products transfusions, cardiac surgery with its related complications, and checking eligibility to heart 


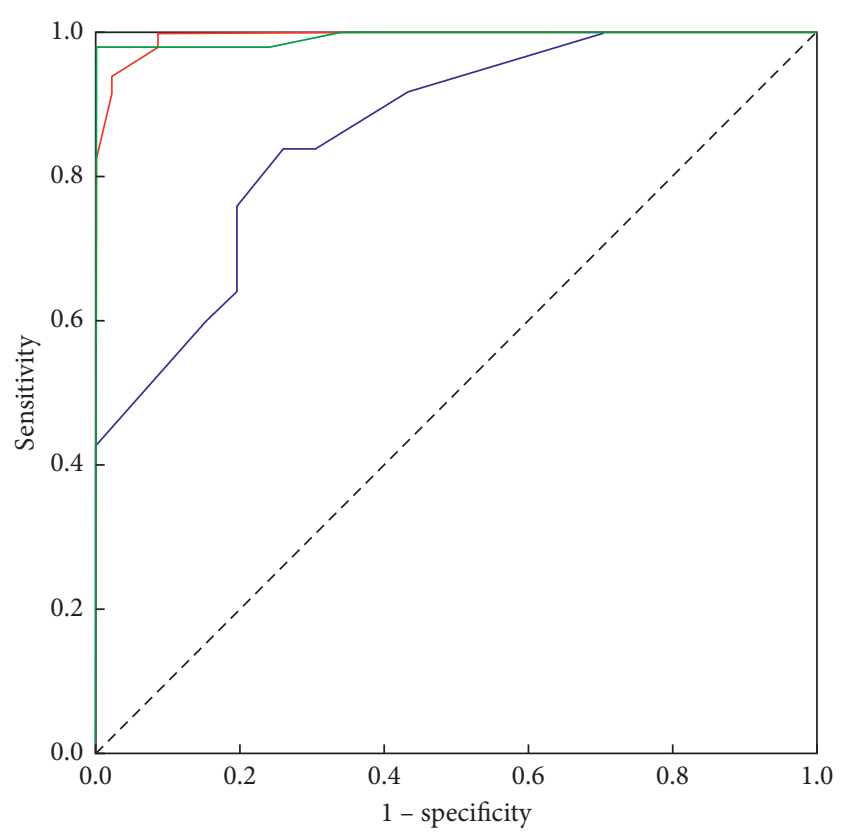

Source of the curve

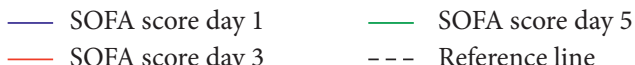

FIGURE 2: ROC of SOFA scores in differentiating mortality.

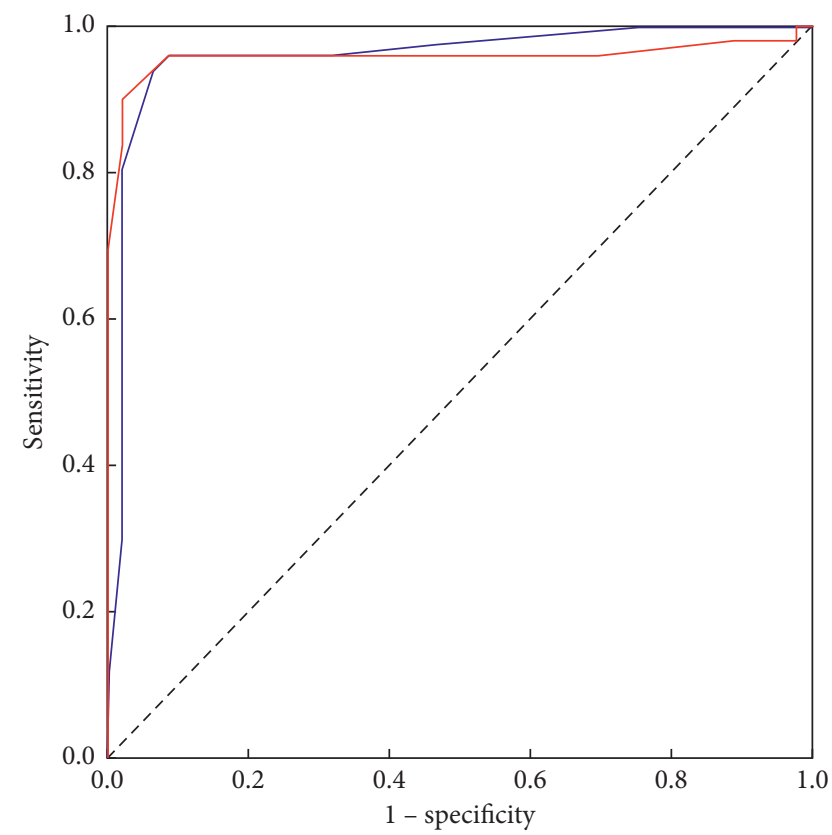

Source of the curve

$-\triangle 1$ SOFA

$-\triangle 2$ SOFA

- - - Reference line

FIgURE 3: ROC of $\Delta 1$ SOFA and $\Delta 2$ SOFA in differentiating mortality.

transplantation or end-of-life decision-making. So we need to score our patients for early decision-making. We used SOFA score for its simplicity and found that initial SOFA
TABLE 7: Multivariable regression analysis of hospital mortality of patients with cardiogenic shock supported with VA-ECMO.

\begin{tabular}{lccc}
\hline Studied variables & $p$ value & OR & $95 \%$ CI for OR \\
\hline Central VA-ECMO & 0.248 & 3.102 & $0.455-21.154$ \\
Hyperlactatemia & 0.040 & 1.388 & $1.015-1.898$ \\
Haemodialysis & 0.712 & 1.614 & $0.127-20.473$ \\
$\Delta$ 1 SOFA (3-1) & $<0.001$ & 2.506 & $1.681-3.735$ \\
GI bleeding & 0.917 & 0.891 & $0.102-7.815$ \\
ICH & 0.424 & 14.585 & $0.020-204.51$ \\
\hline
\end{tabular}

assessment was a good predictor of hospital mortality (AUROC: 0.862). Furthermore, increase in SOFA score at the third and fifth days were associated with hospital mortality, while decreasing SOFA score occurred in the survivors. Increased SOFA $\geq 2$ was an independent predictor of hospital mortality (AUROC: 0.958). Schmidt et al. assessed his studied patients with SOFA and APACHE II and III scores at ECMO initiation only and found that the 3 scoring systems were higher in the nonsurvivors, but that study did not follow the patients during VA-ECMO support, and the AUROC was $0.79,058$, and 0.59 for SOFA, APACHE II, and APACHE III, respectively [18].

Chen et al. developed ROC curves to predict 90-day mortality for VA-ECMO patients, the AUROC was 0.65, 0.73 , and 0.83 for SOFA, SAVE, and modified SAVE scores at ECMO initiation and that study did not follow-up scoring [27].

The haemodynamics deterioration with cardiogenic shock and resuscitation with ECMO support affects the multiorgan functions that change over time and with the efficiency of resuscitation. So we calculated the SOFA score during the first few days of ECMO support and concluded that increasing SOFA after 48 hours of ECMO support is an independent predictor of hospital mortality.

Ferreira et al. demonstrated the efficacy SOFA scoring of critically ill patients without ECMO support at medicosurgical ICU during the first 96 hours of ICU admission. That study showed the trend of SOFA during the first 48 hours of ICU stay being a sensitive indicator of outcome regardless of the initial SOFA [16].

Jentzer et al. recently studied SOFA scoring of critical patients without ECMO in cardiac ICU and the relation of increased SOFA with increased mortality and the lower long-term survival for survivors with high initial SOFA [30]. Recently, SOFA was used to predict the occurrence of acute cerebral strokes in patients supported with VA-ECMO with a good correlation [31].

Finally, VA-ECMO support is associated with frequent morbidities and high mortality. Using SOFA scoring is a simple way to assess and follow VA-ECMO supported adult patients and help in decision-making.

\section{Conclusion}

The use of VA-ECMO in adult patients with cardiogenic shock is still associated with high mortality. Serial evaluation of those patients with SOFA score during first few days of ECMO support is a good predictor of hospital mortality. 
Increase in SOFA score after 48 hours and hyperlactataemia are significantly associated with increased hospital mortality.

\section{Abbreviations}

ACHD: Adult congenital heart disease

ACT: $\quad$ Activated clotting time

AKI: $\quad$ Acute kidney injury

AF: $\quad$ Atrial fibrillation

APACHE: Acute physiology, age, and chronic health evaluation

aPTT: $\quad$ Activated partial thromboplastin time

BMI: $\quad$ Body mass index

CABG: Coronary artery bypass grafting

CKD: $\quad$ Chronic kidney disease

CPB: $\quad$ Cardiopulmonary bypass

DM: $\quad$ Diabetes mellitus

EF: $\quad$ Ejection fraction

GI Gastrointestinal bleeding

bleeding:

IABP: Intra-aortic balloon pump

ICH: Intracerebral haemorrhage

INR: International normalized ratio

LVAD: Left ventricular assist device

ROC: $\quad$ Receiver operating characteristic

SOFA: Sequential organ failure assessment

OR: $\quad$ Odds ratio

VA- Venoarterial extracorporeal membrane

ECMO: oxygenation.

\section{Data Availability}

The data used to support the findings of the study are available from the corresponding author on request.

\section{Additional Points}

Our work was a single center retrospective study with a relatively limited number of patients. It needs further larger prospective validation studies.

\section{Conflicts of Interest}

The authors declare that there are no conflicts of interest.

\section{References}

[1] Y. Asaumi, S. Yasuda, I. Morii et al., "Favourable clinical outcome in patients with cardiogenic shock due to fulminant myocarditis supported by percutaneous extracorporeal membrane oxygenation," European Heart Journal, vol. 26, no. 20, pp. 2185-2192, 2005.

[2] K.-H. Hsu, N.-H. Chi, H.-Y. Yu et al., "Extracorporeal membranous oxygenation support for acute fulminant myocarditis: analysis of a single center's experience," European Journal of Cardio-Thoracic Surgery: Official Journal of the European Association for Cardio-Thoracic Surgery, vol. 40, no. 3, pp. 682-688, 2011.

[3] N. G. Smedira, N. Moazami, C. M. Golding et al., "Clinical experience with 202 adults receiving extracorporeal membrane oxygenation for cardiac failure: survival at five years,"
The Journal of Thoracic and Cardiovascular Surgery, vol. 122, no. 1, pp. 92-102, 2001.

[4] H. Takayama, L. Truby, M. Koekort et al., "Clinical outcome of mechanical circulatory support for refractory cardiogenic shock in the current era," The Journal of Heart and Lung Transplantation, vol. 32, no. 1, pp. 106-111, 2013.

[5] G. H. L. Tang, R. Malekan, M. Kai, S. L. Lansman, and D. Spielvogel, "Peripheral venoarterial extracorporeal membrane oxygenation improves survival in myocardial infarction with cardiogenic shock," The Journal of Thoracic and Cardiovascular Surgery, vol. 145, no. 3, pp. e32-e33, 2013.

[6] A. Combes, P. Leprince, C.-E. Luyt et al., "Outcomes and longterm quality-of-life of patients supported by extracorporeal membrane oxygenation for refractory cardiogenic shock," Critical Care Medicine, vol. 36, no. 5, pp. 1404-1411, 2008.

[7] S. Beurtheret, P. Mordant, X. Paoletti et al., "Emergency circulatory support in refractory cardiogenic shock patients in remote institutions: a pilot study (the cardiac-RESCUE program)," European Heart Journal, vol. 34, no. 2, pp. 112-120, 2013.

[8] S.-Y. Chung, J.-J. Sheu, Y.-J. Lin et al., "Outcome of patients with profound cardiogenic shock after cardiopulmonary resuscitation and prompt extracorporeal membrane oxygenation support," Circulation Journal, vol. 76, no. 6, pp. 1385-1392, 2012.

[9] J. Wang, J. Han, Y. Jia et al., "Early and intermediate results of rescue extracorporeal membrane oxygenation in adult cardiogenic shock," The Annals of Thoracic Surgery, vol. 88, no. 6, pp. 1897-1903, 2009.

[10] A. J. Rastan, A. Dege, M. Mohr et al., "Early and late outcomes of 517 consecutive adult patients treated with extracorporeal membrane oxygenation for refractory postcardiotomy cardiogenic shock," The Journal of Thoracic and Cardiovascular Surgery, vol. 139, no. 2, pp. 302-311, 2010.

[11] F. Yang, D. Hou, J. Wang et al., "Vascular complications in adult postcardiotomy cardiogenic shock patients receiving venoarterial extracorporeal membrane oxygenation," Annals of Intensive Care, vol. 8, no. 1, p. 72, 2018.

[12] C.-H. Chang, H.-C. Chen, J. L. Caffrey et al., "Survival analysis after extracorporeal membrane oxygenation in critically ill adults: a nationwide cohort study," Circulation, vol. 133, no. 24, pp. 2423-2433, 2016.

[13] D. Abrams, A. Combes, and D. Brodie, "Extracorporeal membrane oxygenation in cardiopulmonary disease in adults," Journal of the American College of Cardiology, vol. 63, no. 25, pp. 2769-2778, 2014.

[14] D. Abrams, A. R. Garan, A. R. Garan et al., "Position paper for the organization of ECMO programs for cardiac failure in adults," Intensive Care Medicine, vol. 44, no. 6, pp. 717-729, 2018.

[15] J.-L. Vincent, R. Moreno, J. Takala et al., "The SOFA (sepsisrelated organ failure assessment) score to describe organ dysfunction/failure," Intensive Care Medicine, vol. 22, no. 7, pp. 707-710, 1996.

[16] F. L. Ferreira, D. P. Bota, A. Bross, C. Melot, and J. L. Vincent, "Serial evaluation of the SOFA score to predict outcome in critically ill patients," JAMA, vol. 286, no. 14, pp. 1754-1758, 2001.

[17] S. Lambden, P. Laterre, M. Levy, and B. Francois, "The SOFA score-development, utility and challenges of accurate assessment in clinical trials," Critical Care, vol. 23, p. 374, 2019.

[18] M. Schmidt, A. Burrell, L. Roberts et al., "Predicting survival after ECMO for refractory cardiogenic shock: the survival 
after veno-arterial-ECMO (SAVE)-score," European Heart Journal, vol. 36, no. 33, pp. 2246-2256, 2015.

[19] B. G. Maxwell, A. J. Powers, A. Y. Sheikh, P. H. U. Lee, R. L. Lobato, and J. K. Wong, "Resource use trends in extracorporeal membrane oxygenation in adults: an analysis of the nationwide inpatient sample 1998-2009," The Journal of Thoracic and Cardiovascular Surgery, vol. 148, no. 2, pp. 416-421, 2014.

[20] R. El Sibai, B. Rana, and M. El Sayed, "ECMO use and mortality in adult patients with cardiogenic shock: a retrospective observational study in U.S. hospitals," BMC Emergency Medicine, vol. 18, no. 1, p. 20, 2018.

[21] S. Aso, H. Matsui, K. Fushimi, and H. Yasunaga, "In-hospital mortality and successful weaning from venoarterial extracorporeal membrane oxygenation: analysis of 5,263 patients using a national inpatient database in Japan," Critical Care, vol. 20 , no. 1, p. 80, 2016.

[22] L. Wang, F. Yang, X. Wang et al., "Predicting mortality in patients undergoing VA-ECMO after coronary artery bypass grafting: the REMEMBER score," Critical Care, vol. 23, no. 1, 2019.

[23] G. Mariscalco, A. Salsano, A. Fiore et al., "Peripheral versus central extracorporeal membrane oxygenation for postcardiotomy shock: multicenter registry, systematic review and meta-analysis," The Journal of Thoracic and Cardiovascular Surgery, 2019.

[24] I. Djordjevic, K. Eghbalzadeh, A. Sabashnikov et al., "Central vs peripheral venoarterial ECMO in postcardiotomy cardiogenic shock," Journal of Cardiac Surgery, vol. 35, no. 5, pp. 1037-1042, 2020.

[25] L. Rupprecht, B. Flörchinger, S. Schopka et al., "Cardiac decompression on extracorporeal life support: a review and discussion of the literature," ASAIO Journal, vol. 59, no. 6, pp. 547-553, 2013.

[26] A. Gass, C. Palaniswamy, W. S. Aronow et al., "Peripheral venoarterial extracorporeal membrane oxygenation in combination with intra-aortic balloon counterpulsation in patients with cardiovascular compromise," Cardiology, vol. 129, no. 3, pp. 137-143, 2014.

[27] W.-C. Chen, K.-Y. Huang, C.-W. Yao et al., "The modified SAVE score: predicting survival using urgent veno-arterial extracorporeal membrane oxygenation within 24 hours of arrival at the emergency department," Critical Care, vol. 20, no. 1 , p. 336,2016

[28] M. S. Englehart and M. A. Schreiber, "Measurement of acidbase resuscitation endpoints: lactate, base deficit, bicarbonate or what?" Current Opinion in Critical Care, vol. 12, no. 6, pp. 569-574, 2006.

[29] P. Perez, A. Kimmoun, V. Blime, and B. Levy, "Increasing mean arterial pressure in cardiogenic shock secondary to myocardial infarction: effects on hemodynamics and tissue oxygenation," Shock, vol. 41, no. 4, pp. 269-274, 2014.

[30] J. C. Jentzer, C. Bennett, B. M. Wiley et al., "Predictive value of the sequential organ failure assessment score for mortality in a contemporary cardiac intensive care unit population," Journal of the American Heart Association, vol. 7, no. 6, Article ID e008169, 2018.

[31] M. Laimoud and W. Ahmed, "Acute neurological complications in adult patients with cardiogenic shock on venoarterial extracorporeal membrane oxygenation support," The Egyptian Heart Journal, vol. 72, no. 1, 2020. 